\title{
The Galaxy and its stellar halo - insights from a hybrid cosmological approach
}

\author{
Gabriella De Lucia ${ }^{1}$ and Amina Helmi ${ }^{2}$ \\ ${ }^{1}$ Max-Planck-Institut für Astrophysik, \\ Karl-Schwarzschild-Str. 1, D-85748 Garching, Germany \\ email: gdelucia@mpa-garching.mpg.de \\ ${ }^{2}$ Kapteyn Astronomical Institute, University of Groningen, \\ P.O. Box 800, 9700 AV Groningen, Netherlands \\ email: ahelmi@astro.rug.nl
}

\begin{abstract}
We use a series of high-resolution N-body simulations of a 'Milky-Way' halo, coupled to semi-analytic techniques, to study the formation of our own Galaxy and of its stellar halo. Our model Milky Way galaxy is a relatively young system whose physical properties are in quite good agreement with observational determinations. In our model, the stellar halo is mainly formed from a few massive satellites accreted early on during the galaxy's lifetime. The stars in the halo do not exhibit any metallicity gradient, but higher metallicity stars are more centrally concentrated than stars with lower abundances. This is due to the fact that the most massive satellites contributing to the stellar halo are also more metal rich, and dynamical friction drags them closer to the inner regions of the host halo.
\end{abstract}

Keywords. Methods: N-body simulations, Galaxy: evolution, Galaxy: formation, Galaxy: halo

\section{Introduction}

Our own galaxy - the Milky Way - is a fairly large spiral galaxy consisting of four main stellar components: (1) the thin disk, that contains most of the stars with a wide range of ages and on high angular momentum orbits; (2) the thick disk, that contains about 10-20 per cent of the mass in the thin disk and whose stars are on average older and have lower metallicity than those in the thin disk; (3) the bulge, which contains old and metal rich stars on low angular momentum orbits; and (4) the stellar halo which contains only a few per cent of the total stellar mass and whose stars are old and metal poor and reside on low angular momentum orbits.

While the Milky Way is only one Galaxy, it is the one that we can study in unique detail. Over the past years, accurate measurements of ages, metallicities and kinematics have been collected for a large number of individual stars, and much larger datasets will become available in the next future thanks to a number of ongoing and planned astrometric, photometric and spectroscopic surveys. This wealth of detailed and high-quality observational data provides an important benchmark for current theories of galaxy formation and evolution.

In the following, we outline the main results of a recent study of the formation of the Milky Way and of its stellar halo in the context of a hybrid cosmological approach which combines high-resolution simulations of a 'Milky Way' halo with semi-analytic methods. We refer to De Lucia \& Helmi (2008) for a more detailed description of our method and of our results. 


\section{The simulations and the galaxy formation model}

We use the re-simulations of a 'Milky-Way' halo (the GA series) described in Stoehr et al. (2002) and Stoehr et al. (2003), with an underlying flat $\Lambda$-dominated CDM cosmological model. The candidate halo for re-simulations was selected from an intermediateresolution simulation (particle mass $\sim 10^{8} \mathrm{M}_{\odot}$ ) as a relatively isolated halo which suffered its last major merger at $z>2$. The same halo was then re-simulated at four progressively higher resolution simulations with particle mass $\sim 1.7 \times 10^{8}(\mathrm{GA} 0), \sim 1.8 \times 10^{7}$ $(\mathrm{GA} 1), \sim 1.9 \times 10^{6}(\mathrm{GA} 2)$, and $\sim 2.1 \times 10^{5} \mathrm{M}_{\odot}$ (GA3). Simulation data were stored in 108 outputs from $z=37.6$ to $z=0$, and for each simulation output we constructed group catalogues (using a standard friends-of-friends algorithm) and substructure catalogues (using the SUBFIND algorithm developed by Springel et al. 2001). Substructure catalogues were then used to construct merger history trees for all self-bound haloes as described in Springel et al. (2005) and De Lucia \& Blaizot (2007). Finally, these merger trees were used as input for our semi-analytic model of galaxy formation.

\section{Physical properties and metallicity distributions}

Fig. 1 shows the evolution of different mass components for the model Milky Way galaxies in the four simulations used in our study (lines of different colours). The histories shown in the different panels have been obtained by linking the galaxy at each time-step to the progenitor with the largest stellar mass. Fig. 1 shows that approximately half of the final mass in the dark matter halo is already in place (in the main progenitor) at $z \sim 1.2$ (panel a) while about half of the final total stellar mass is only in place at $z \sim 0.8$ (panel

redshift

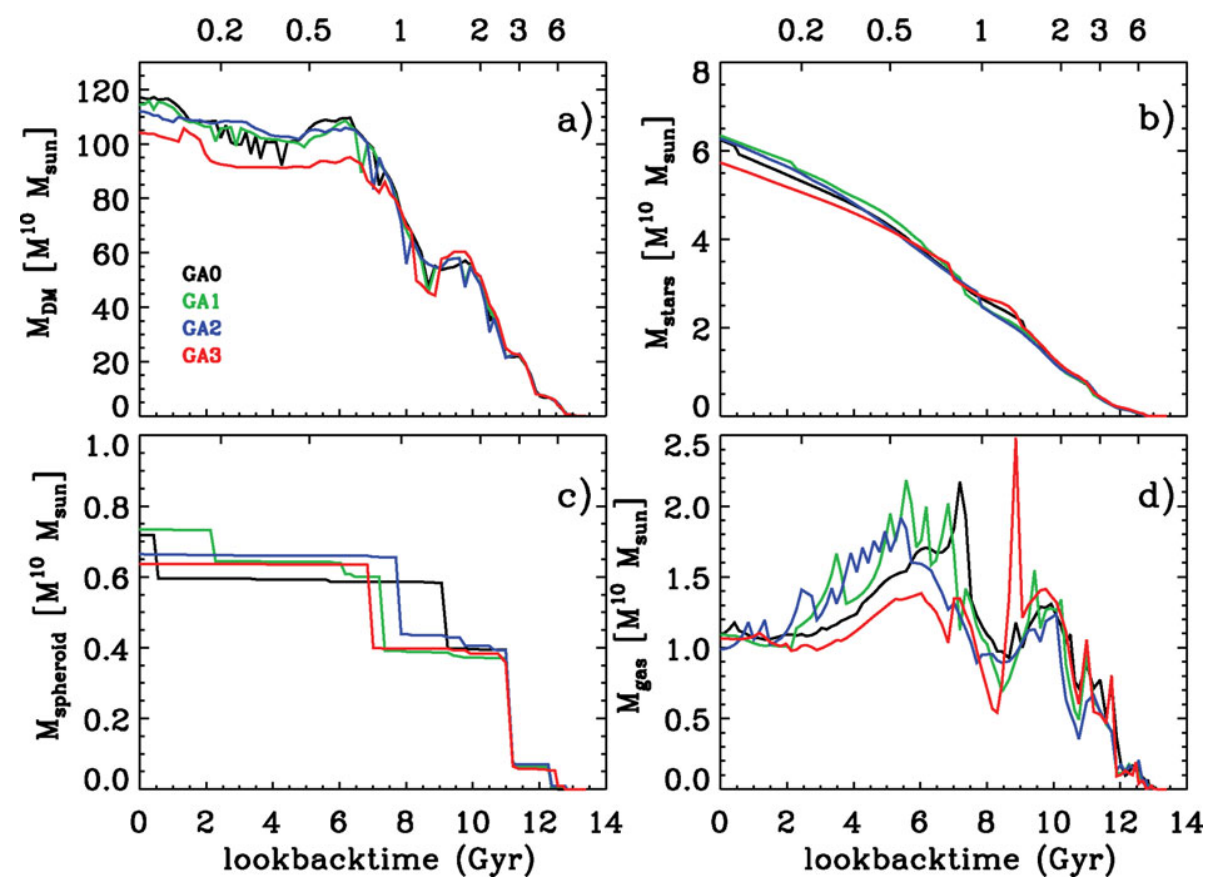

Figure 1. Evolution of the dark matter mass (panel a), total stellar mass (panel b), spheroid mass (panel c), and cold gas mass (panel d) for the model Milky Way in the four simulations used in our study (different colours). 
b). About 20 per cent of this stellar mass is already in a spheroidal component (panel c). The mass of the spheroidal component grows in discrete steps as a consequence of our assumption that it grows during mergers and disk instability episodes, and approximately half of its final mass is already in a spheroidal component at $z \sim 2.5$. In contrast, the cold gas mass varies much more gradually.

Interestingly, the model produces consistent evolutions for all four simulations used in our study, despite the large increase in numerical resolution. Some panels (e.g. panel b) do not show perfect convergence, due to the lack of complete convergence in the N-body simulations (see panel a). Fig. 1 also shows that the total stellar mass of our model Galaxy $\left(6 \times 10^{10} \mathrm{M}_{\odot}\right)$ is is very good agreement with the estimated value $\sim 5-8 \times 10^{10} \mathrm{M}_{\odot}$. The mass of the spheroidal component is instead slightly lower than the observed value (assumed to be about 25 per cent of the disk stellar mass), while our fiducial model gives a gas mass which is about twice the estimated value.
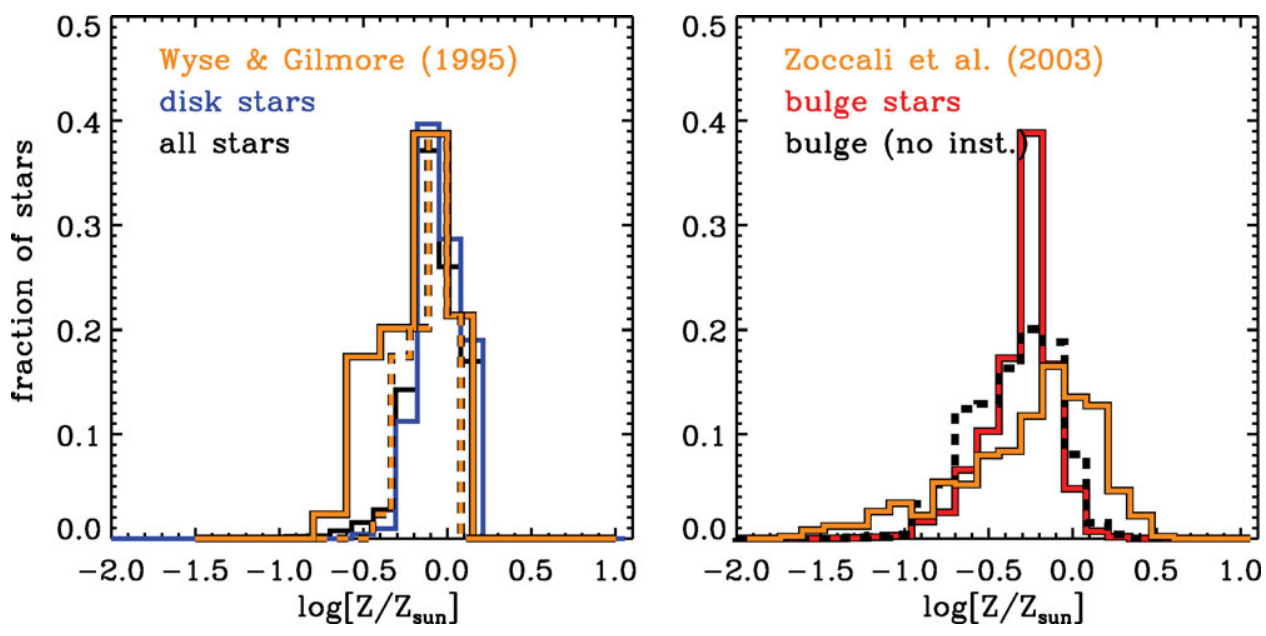

Figure 2. Metallicity distribution for stars in the disk (blue histogram in the left panel) and spheroid (red histogram in the right panel) of the model Milky Way from the highest resolution simulation in our study. The solid black histogram in the left panel shows the metallicity distribution for all stars in the model galaxy, while the dashed black histogram in the right panel shows the metallicity distribution of stars in the spheroidal component for our fiducial model if spheroid growth through disk instability is suppressed. The solid orange histograms show observational measurements by Wyse \& Gilmore (1995, left panel) and Zoccali et al. (2003, right panel). The dashed orange histogram in the left panel has been obtained converting the $[\mathrm{Fe} / \mathrm{H}]$ scale of the original distribution by Wyse \& Gilmore into an $[\mathrm{O} / \mathrm{H}]$ scale by using the observed $[\mathrm{O} / \mathrm{H}]-[\mathrm{Fe} / \mathrm{H}]$ relation for thin disk stars by Bensby, Feltzing \& Lundström (2004).

Fig. 2 shows the metallicity distributions of the stars in the disk and spheroid of our model Milky Way from the highest resolution simulation used in our study. The left panel shows the metallicity distribution of all stars (black) and of the stars in the disk (blue) compared to the observational measurements by Wyse \& Gilmore (1995). The right panel shows the metallicity distribution of the spheroid stars in our fiducial model (red) and in a model where the disk instability channel is switched off (dashed black). Model results are compared to observational measurements by Zoccali et al. (2003). The metallicity distribution of disk stars in our model peaks at approximately the same value as observed, but it exhibits a deficiency of low metallicity stars. When comparing model results and observational measurements, however, two factors should be considered: (1) the observational measurements have some uncertainties $(\sim 0.2 \mathrm{dex})$ which tend 
to broaden the true underlying distributions; (2) the observational measurements provide iron distributions, and iron is not well described by our model that adopts an instantaneous recycling approximation. In order to show the importance of this second caveat we have converted the measured $[\mathrm{Fe} / \mathrm{H}]$ into $[\mathrm{O} / \mathrm{H}]$ using a linear relation, obtained by fitting data for thin disk stars from Bensby, Feltzing \& Lundström (2004). The result of this conversion is shown by the dashed orange histogram in the left panel of Fig. 2. The observed $[\mathrm{O} / \mathrm{H}]$ metallicity distribution is now much closer to the modelled $\log \left[\mathrm{Z} / \mathrm{Z}_{\odot}\right]$ distribution. The same caveats applies to the comparison shown in the right panel, which indicates that our model spheroid is significantly less metal rich than the observed Galactic bulge.

\section{The stellar halo}

In order to study the structure and metallicity distribution of the stellar halo, we assume that it builds up from the cores of the satellite galaxies that merged with the Milky Way over its lifetime. The stars that end up in the stellar halo are identified by tracing back all galaxies that merged with the Milky Way progenitor, until they are central galaxies of their own halo. We select then a fixed fraction (10 per cent for the results shown in the following) of the most bound particles of their parent haloes, and tag them with the mean metallicity of the central galaxies (for details, see De Lucia \& Helmi 2008).
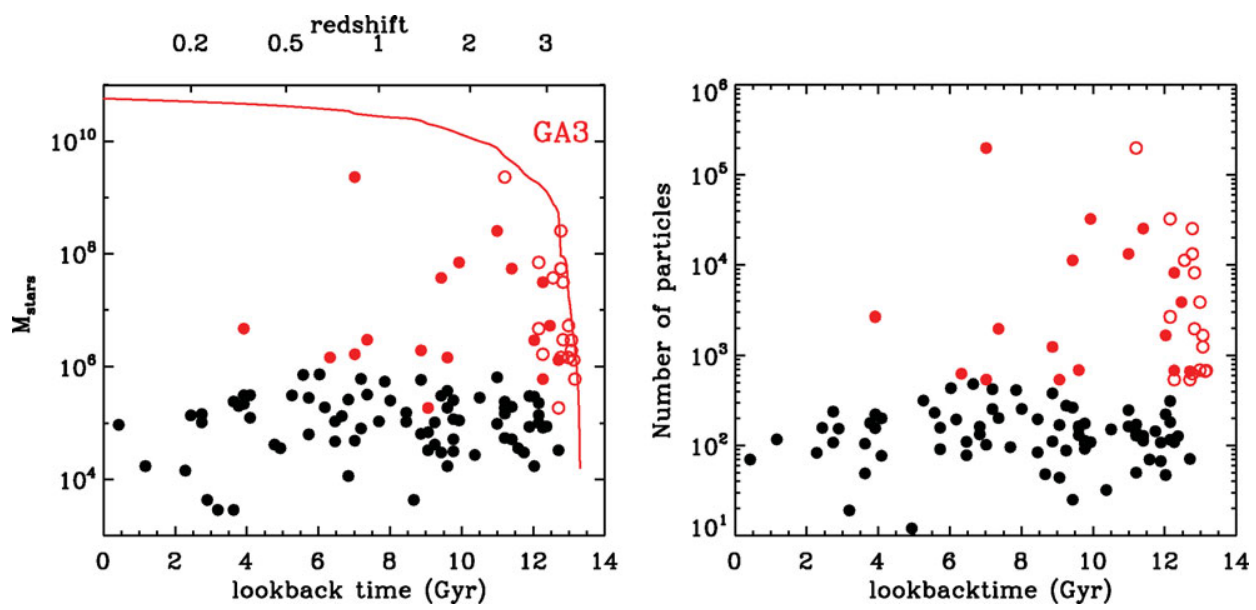

Figure 3. Stellar mass (left panel) of the galaxies contributing to the stellar halo, as a function of the lookback time of galaxy's merger. The right panel shows the number of particles associated to the dark matter haloes at the time of accretion. Red symbols correspond to objects with more than 500 particles. Open symbols correspond to red filled circles but are plotted as a function of the time of accretion.

Fig. 4 shows the stellar mass of the galaxies contributing to the stellar halo as a function of the lookback time of the galaxy's merger (left panel), and the number of particles associated to the dark matter haloes at the time of accretion (right panel). Most of the accreted galaxies lie in quite small haloes and only a handful of them are attached to relatively more massive systems, which are accreted early on during the galaxy's lifetime. These are the galaxies that contribute most to the build-up of the stellar halo. The results illustrated in Fig. 4 are in good agreement with those by Font et al. (2006) who combined 

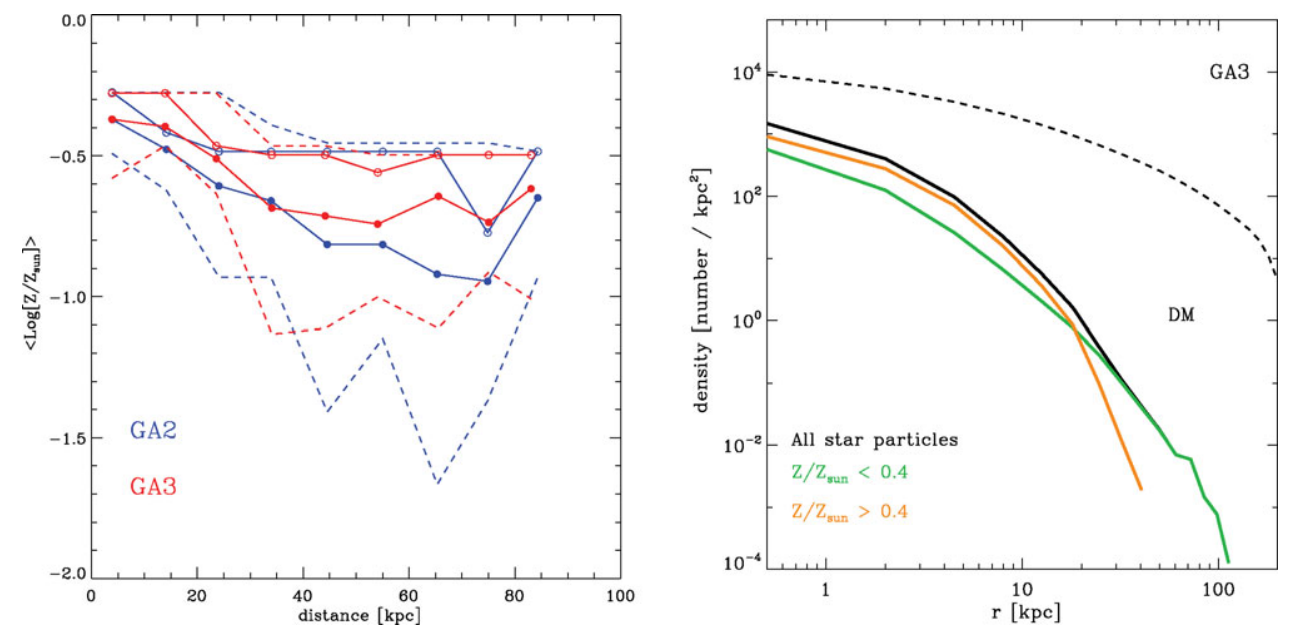

Figure 4. Left panel: Mean (filled circles) and median (empty circles) metallicity of star particles as a function of the distance from the most bound particle in the Milky Way halo, for the simulations GA2 (blue) and GA3 (red). Dashed lines correspond to the 15th and 85th percentiles of the distribution. Right panel: Projected density profile of the stellar halo (solid black line) and of the dark matter halo (dashed black line) for the simulation GA3. The solid green and orange lines show the projected density profiles for star particles with metallicity smaller and larger than $0.4 Z_{\odot}$ respectively.

mass accretion histories of galaxy-size haloes with a chemical evolution model for each accreted satellites to study the formation of the stellar halo.

Fig. 4 shows the metallicity of star particles as a function of the distance from the most bound particle in the Milky Way halo for the simulations GA2 (blue) and GA3 (red). For both simulations, the mean metallicity decreases from $\log \left[\mathrm{Z} / \mathrm{Z}_{\odot}\right] \sim-0.4$ at the centre to $\sim-0.8$ at a distance of $\sim 40 \mathrm{kpc}$. The median and upper $85 \mathrm{th}$ percentile of both distributions are approximately flat around $\sim-0.5$. Note that the metallicity of our stellar halo is higher than what is known for the Galactic halo near the Sun. We note also that both distributions are dominated in number by star particles associated to one or a few accreted galaxies with relatively high metallicity (hence the flat behaviour of the median and upper percentile of the distribution). The lower percentile declines with increasing distance from the centre, suggesting that the inner region is largely dominated by high-metallicity stars while the contribution from lower metallicity stars becomes more important moving to the outer regions. This is shown more explicitely in the right panel of Fig. 4 which shows the projected density profile of the stellar halo (black) for the simulation GA3. The solid orange and green lines in this panel show the projected profiles of star particles with metallicity larger and smaller than $0.4 Z_{\odot}$ respectively. High metallicity stars are more centrally concentrated than stars with lower abundances, suggesting that the probability of observing low-metallicity stars increases at larger distances from the Galactic centre $(\gtrsim 10-20 \mathrm{kpc})$, where the contribution from the inner more metal-rich stars is less dominant. Interestingly, this result appears to be in qualitative agreement with recent measurements by Carollo et al. (2007).

In our model, the 'dual' nature of the stellar halo originates from a correlation between the stellar metallicity and the stellar mass of accreted galaxies. Since the most massive galaxies decay through dynamical friction to the inner regions of the halo, this is where higher metallicity stars will be found preferentially. 


\section{Conclusions}

We have combined high-resolution resimulations of a 'Milky Way' halo with semianalytic techniques to study the formation of our own Galaxy and of its stellar halo. The galaxy formation model used in our study has been used in a number of previous studies and has been shown to provide a reasonable agreement with a large number of observational data both in the local Universe and at higher redshifts (De Lucia \& Helmi 2008 and references therein). Our study demonstrates that the same model is able to reproduce quite well the observed physical properties of our own Galaxy. The agreement is not perfect: our model Galaxy contains about twice the gas observed in the Milky Way, and the model bulge is slightly less massive and substantially less metal rich than the Galactic bulge. A detailed comparison between model results and observational measurements of metallicity distributions is complicated by the use of an instantaneous recycling approximation which is not appropriate for the iron-peak elements, mainly produced by supernovae Type Ia. Relaxing of this approximation in future work, will allow us to carry out a more detailed comparison with observed chemical compositions, and to establish similarities and differences between present-day satellites and the building blocks of the stellar halo.

Our model stellar halo is made up of very old stars (older than $\sim 11$ Gyr) with low metallicity, although higher than what is known for the stellar halo of our Galaxy. Most of the stars in the halo are contributed by a few relatively massive satellites accreted early on during the galaxy's lifetime. The building blocks of the stellar halo lie on a well defined mass-metallicity relation. Since the most massive galaxies are dragged closer to the inner regions of the halo by dynamical friction, this produces a stronger concentration of more metal rich stars, in qualitative agreement with recent observational measurements. The numerical resolution of our simulations is too low for the study of spatially and kinematically coherent structures in model stellar halo. Higher resolution simulations are needed for this kind of study.

\section{References}

Bensby, T., Feltzing, S., \& Lundström, I. 2004, A\&A, 415, 155

Carollo, D., Beers, T. C., Lee, Y. S., Chiba, M., Norris, J. E., Wilhelm, R., Silvarani, T., Marsteller, B., Munn, J. A., Bailer-Jones, C. A. L., Fiorentin, P. R., \& York, D. G. 2007, Nature, 450, 1020

De Lucia, G. \& Blaizot, J. 2007, MNRAS, 375, 2

De Lucia, G. \& Helmi, A. 2008, MNRAS submitted, arXiv:0804.2465

Font, A. S., Johnston K. V., Bullock J. S., \& Robertson, B. E. 2006, ApJ, 638, 585

Springel, V., White, S. D. M., Tormen, G., \& Kauffmann, G. 2001, MNRAS, 328, 726

Springel, V., White, S. D. M., Jenkins, A., Frenk, C. S., Yoshida, N., Gao, L., Navarro, J., Thacker, R., Croton, D., Helly, J., Peacock, J. A., Cole, S., Thomas, P., Couchman, H., Evrard, A., Corlberg, J., \& Pearce, F. 2005, Nature, 435, 629

Stoehr, F., White, S. D. M., Tormen, G., \& Springel, V. 2002, MNRAS, 335, L84

Stoehr, F., White, S. D. M., Springel, V., Tormen, G., \& Yoshida, N. 2003, MNRAS, 345, 1313 Wyse, R. F. G. \& Gilmore, G. 1995, AJ, 110, 2771

Zoccali, M., Renzini, A., Ortolani, S., Greggio, L., Saviane, I., Cassisi, S., Rejkuba, M., Barbuy, B., Rich, R. M., \& Bica, E. 2003, A\&BA, 399, 931 